\title{
Nanostructured coatings, surfaces and films
}

\author{
Kock-Yee Law \\ Research and Innovative Solutions, Penfield, NY, USA
}

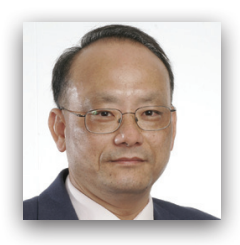

Nanotechnology is a descriptor of a frontier for the understanding and control of matter at dimensions of roughly from 1 to $100 \mathrm{~nm}$, where unique phenomena enable novel applications. Nanotechnology encompasses nanoscale science, technology, engineering and manufacturing, involving manipulating and interactions of matters, measuring and modeling them at the nanometer scale. At this length level, due to the quantum confinement effect and/or the extraordinary aspect ratio and surface area, the physical, chemical and biological properties of materials differ in fundamental and valuable ways from the properties of individual atoms or bulk matter. One of the intersection points between nanotechnology and physical science is surface. Surface is a very important branch of science that touches all facets of our lives. Studies of wettability, repellency, spreading, adhesion and lubrication, are not only important scientifically but also of practical significance to a variety of applications, such as coating, painting, printing, airplane deicing, ship antifouling, gas and fuel transportations, microfluidic devices, anticorrosion in metals, contamination management and controls, cleaning and washing in our daily live and so forth. In 1936, Wenzel ${ }^{1}$ reported that roughness in surface enhances liquid wettability when the contact angle of the flat surface $\left(\theta_{\mathrm{Y}}\right)$ is $<90^{\circ}$. However, the surface will resist wetting when $\theta_{\mathrm{Y}}>90^{\circ}$. The contact angle on the rough surface $\left(\theta_{\mathrm{w}}\right)$ is given by the Wenzel equation:

$$
\text { 1. } \cos \theta_{\mathrm{W}}=r \cdot \cos \theta_{\mathrm{Y}}
$$

where $r$ is the ratio between the surface area of the rough surface and the flat surface.

Cassie-Baxter $(\mathrm{CB})^{2}$ later found that when $r$ is large, the increase in surface roughness traps air between the liquid and the solid surface. The $\mathrm{CB}$ contact angle $\left(\theta_{\mathrm{CB}}\right)$ at the composite interface is given by

$$
\text { 2. } \cos \theta_{\mathbf{C B}}=r_{f} f \cdot \cos \theta_{\mathbf{Y}}+f-1
$$

where $f$ is the area fraction of the projected wet area and $\mathbf{r}_{f}$ is the solid area fraction.

As our understanding in wetting is advancing, there have been discussions and debates about the validity and range of applicability of these two equations. ${ }^{3-9}$ Nevertheless, these two articles are classic, and they described the two basic states of a liquid droplet on a rough surface. Studies of liquid-solid interactions on rough surfaces appear to go into dormancy until 1997 when Barthlott and Neinhuis published their famous Planta article about the self-cleaning of the lotus leaf. ${ }^{10}$ The multiscale texture on the lotus leaf surface was shown to be responsible for the superhydrophobic, self-cleaning effect, where droplet of water beads up with an extremely high contact angle and roll off upon very slight tilting. ${ }^{11-14}$ The lotus effect appeared to inspire researchers around the world and activities on superhydrophobicity and later superoleophobicity have been very rigorous since. Figure 1 plots the number of English publications studying superhydrophobicity and superoleophobicity since 1990 from a brief SciFinder search. Thousands of articles are published annually in recent years. Research topics range from studies of the fundamental wetting phenomena of plants, insects and animals to the fabrication of artificial micro, nanostructured surfaces that mimic the lotus leaves to the exploration of these surfaces for a variety of applications. It is clear from the plot that research activities on superhydrophobic and superoleophobic surfaces will continue to grow rapidly. As the science related to superhydrophobic and superoleophobic surfaces is maturing, design criteria become better understood, and market opportunities start to open up, technology issues related to the robustness of the surface design and the latitude for large area, largescale manufacturing will need to be addressed. A successful conquer of the latter two issues will be crucial to any product implementation.

The Nanotech Conference organized by Nano Science and Technology Institute is the largest Nanotech meeting annually in the USA. It attracts thousands of participants all over the world each year. I have been an active participant of the "Nanostructured Coatings and Surfaces symposium" since 2009 and became the symposium's (co)organizer since 2011. The symposium has great global appeal, attracting researchers from academia, industry and government labs in four different continents (North American, Europe, Asia and Australia). This special themed issue published by Surface Innovations includes invited speakers in the last two symposia and contributors from Nanotech 2012. The issue features six articles from active researchers in this field and will give readers a snapshot of the research and trend as well as the demographic of the contributors. The first article is a brief review from the laboratories of Dr. A. Tuteja at the University of Michigan and Dr. J. Mabry at the Air Force Research Lab at the Edwards AFB. This article provides a timely 


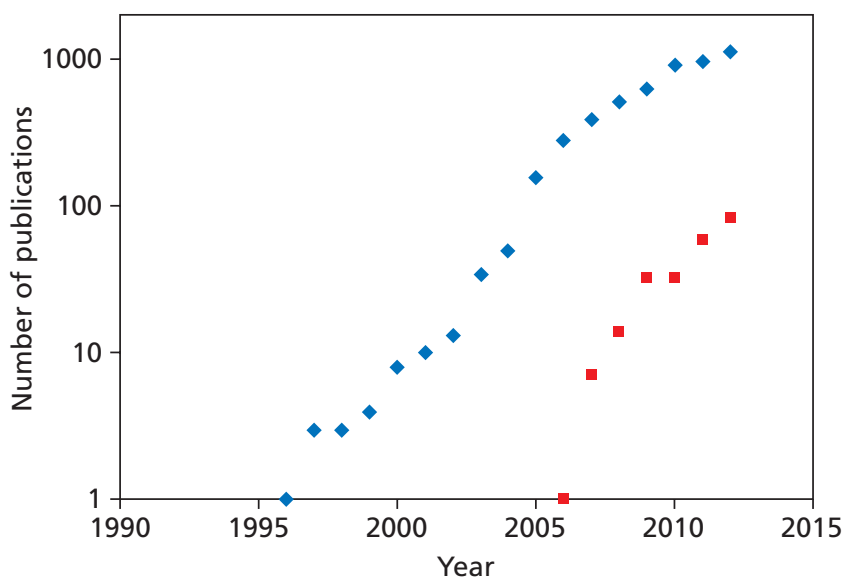

Figure 1. Plot of number of publications on superhydrophobic and superoleophobic surfaces versus time since 1990.

review of the exciting field in superoleophobicity. The design criteria for low hysteresis, super oil-repelling surfaces are presented with a nice description of the trend and outlook at the end. The second contribution is from Dr. K. Varanasi's lab at Massachusetts Institute of Technology. Dr. Varanasi reports the visualization of the movement of the contact line when water droplets are advancing and receding on a superhydrophobic postarray surface. The advancing contact line is shown to glide smoothly over the micro-posts, whereas the receding contact line exhibits stick-slip behavior. This study provides significant insight into the dynamic nature of contact angle hysteresis and the design of superhydrophobic surface with optimal drop shedding. The third article is by Dr. B. Wang of Svaya Nanotechnology, Inc. at Sunnyvale, CA, Dr. Wang's team reports the use of layer-by-layer (LbL) technique to prepare antireflection coating for automobile application. This work is significant; it reports the development of a manufacturable spray process for the LbL coating. Prototypes of the coated films were available for viewing at the Nanotech 2012 conference. In addition, Dr. Wang's team also introduces a sol-gel processing step during manufacturing to enhance the mechanical durability of the antireflection LbL coating. The fourth and fifth contributions report two different methods to prepare superhydrophobic surfaces and coatings. Dr. F. Guittard's team at Nice University (France) reports the electrodeposition of alkyl 3,4-ethylenedioxythiophene to form superhydrophobic films. The effect of alkyl chain length on the microstructure and nanoporosity is presented and discussed. Dr. S. T. Aruna at the National Aerospace Lab in Bangalore, India, shows that superhydrophobic films can be prepared by spraying a coating solution consisting of nanosilica particles and polystyrene. The final article of this themed issue is from Dr. N. Dutta's group at University of South Australia, Australia. One of the potential applications for nanostructured coatings and surfaces is for anticorrosion. In this final chapter, Dr. Dutta's team provides a comprehensive survey of the approaches studied by researchers in this field. This article is highly recommended to new comers and researchers who want to have a quick update on anticorrosion coatings.
In summary, this themed issue provides a snapshot of key research areas and trend in nanostructured coatings and surfaces. On the basis of literature search and participants in the Nanotech conferences, research activities in superhydrophobic and superoleophobic surfaces and their applications are very global and active. Thousands of papers are published annually. Researchers now have a good handle in the basic design criteria and begin to focus on topics that are relevant to productization, such as hysteresis, wetting breakthrough pressure, mechanical robustness and manufacturing issues.

\section{REFERENCES}

1. Wenzel, R. N. Resistance of solid surfaces to wetting by water. Industrial \& Engineering Chemistry Research 1936, 28, 988-994.

2. Cassie, A. B. D.; Baxter, S. Wetting of porous surfaces. Transactions of the Faraday Society 1944, 40, 546-551.

3. Gao, L.; McCarthy, T. J. How Wenzel and Cassie were wrong? Langmuir 2007, 23, 3762-3765.

4. Panchagnula, M. V.; Vedantam, S. Comment on how Wenzel and Cassie were wrong. Langmuir 2007, 23, 13242.

5. Gao, L.; McCarthy, T. J. Reply to "comment on How Wenzel and Cassie were wrong by Gao and McCarthy." Langmuir 2007, 23, 13243.

6. Gao, L.; McCarthy, T. J. An attempt to correct the faulty intuition perpetuated by the Wenzel and Cassie "Laws." Langmuir 2009, 25, 7249-7255.

7. Nosonovsky, M. On the range of applicability of the Wenzel and Cassie equations. Langmuir 2007, 23, 9919-9920.

8. Erbil, H. Y.; Cansoy, C. E. Range of applicability of the Wenzel and Cassie-Baxter equations for superhydrophobic surfaces. Langmuir 2009, 25, 14135-14145.

9. Milne, A. J. B.; Amirfazli, A. The cassie equation: how is it means to be used? Advances in Colloid and Interface Science 2012, 170, 48-55.

10. Barthlott, W.; Neinhuis, C. The purity of sacred lotus or escape from contamination in biological surfaces. Planta 1997, 202, 1-8.

11. Neinhuis, C.; Barthlott, W. Characterization and distribution of water-repellent, self-cleaning plant surfaces. Annals of Botany 1997, 79, 667-677.

12. Cheng, Y. T.; Rodak, D. E.; Wong, C. A.; Hayden, C. A. Effects of micro and nanostructures on the self-cleaning behavior of lotus leaves. Nanotechnology 2006, 17, 1350-1362.

13. Gao, L.; McCarthy, T. J. The "lotus effect" explained: two reasons why two length scales of topography are important. Langmuir 2006, 22, 2966-2967.

14. Bittoun, E.; Marmur, A. The role of multiscale roughness in the lotus effect: is it essential for superhydrophobicity? Langmuir 2012, 28, 13933-13942. 